velocity selector and, after interaction with a sample, the neutron energy is redetermined. The instrument design is dominated by the relatively low flux of neutron beams and this problem is particularly acute when a high-energy resolution is required, for example to probe slow motions in polymer chains. The breakthrough which the spin echo technique represents is that the velocity of the neutron is determined from the Larmor precession of the neutron spin in a magnetic field. In this way the resolution of the energy change of scattering does not depend on the monochromatization of ingoing or outgoing neutron beams, and very small energy changes down to $10^{-8} \mathrm{eV}$ can be measured.

This book, which is based on a workshop at the ILL, is in three parts. The first deals with the method from the point of view of both theory and its practical realization. The discussion is sufficiently detailed for the specialist to appreciate as a review, while at the same time it provides a useful introduction for the intending practitioner.

The non-specialist will probably be more interested in the second part which gives a range of examples. Even though most of the measurements are preliminary, this section well illustrates how improvements in neutron instrumentation generate fresh scientific opportunities. The chapters which are likely to be the best indicators of future research are those on the dynamics of micelle solutions, polymer solutions, spin glass dynamics and superfluid ${ }^{4} \mathrm{He}$, although the reader must beware of the rapidity of developments in these and other fields. The third section deals primarily with future instrumentation; it will serve for some time as food for thought for the experiments of tomorrow. Apart from concluding comments by the editor, there are also interesting chapters on the use of spin echo methods to discriminate against thermal diffuse scattering and on the application of NSE techniques in order to measure phonon line widths.

In sum, then, this is a thoroughly worthwhile contribution to a good series.

B. E. F. Fender is Directeur Adjoint at the Institut Max von Laue-Paul Langevin, Grenoble.

\title{
Reluctant leaf-eaters of Barro Colorado
}

\section{R.D. Martin}

The Foraging Strategy of Howler Monkeys: A Study in Primate Economics. By Katharine Milton. Pp.165. ISBN 0-231-04850-5. (Columbia University Press: 1980.) $\$ 20, £ 13$.

IT IS generally agreed that C.R. Carpenter's 1934 monograph (Comp. Psychol. Monogr. 10, 1-168) on mantled howler monkeys, Alouatta palliata, marked a significant transition in primate field studies, from essentially anecdotal accounts to serious scientific investigations. His study was conducted on the small island of Barro Colorado (BCI), an area of about 1,500 hectares of rainforest isolated during the construction of the Panama Canal in 1914. The Smithsonian Tropical Research Institute has, over a period of several decades, sponsored numerous fundamental studies of the fauna and flora. In the half century since Carpenter's publication, quite a few studies have been conducted on the primate species inhabiting $\mathrm{BCI}$, and the howler monkey population (now numbering over 1,000 individuals) has been re-studied and re-censused at fairly regular intervals. Alouatta palliata therefore counts among the best-known primate species with respect to natural behaviour and ecology.

The first systematic primate field studies were, inevitably, largely concerned with basic data collection and primarily qualitative. As work has progressed, there has been increasing emphasis on quantification of both behavioural and ecological parameters, and most modern studies are specifically designed to answer welldefined questions. There has also been increasing integration of laboratory studies with field work as more specific issues, such as nutritional and toxic properties of natural foodstuffs, have come into focus. All of these modern trends are admirably exemplified in Katharine Milton's The Foraging Strategy of Howler Monkeys, which provides a fine example of the great advances made since Carpenter's pioneering work. Appropriately, Milton's main 14-month field study was also conducted on BCI, on the direct descendants of the monkeys studied by Carpenter.

The howler is one of the largest of the New World monkeys (Ceboidea) and has been widely regarded as the most folivorous. Milton's quantitative, yearround data confirm that, on average, the mantled howler spends more time eating leaves than either fruits or flowers. Yet the howler lacks any obvious specialization of its digestive tract to extract particular benefit from an abundant gut flora in processing the leaves in its diet (viz. breakdown of cell wall material; detoxification). For instance, there is no sacculation of the stomach as is found in the specialized leafeating monkeys of the Old World (Colobinae), and the caecum is not notably enlarged. Using carefully-collected quantitative data on both behaviour and ecology, Milton sets out to define the "foraging strategy" of the howlers and to explain the lack of obvious digestive tract specialization. In a clear and economically written account, she gradually constructs a picture of the mantled howler as a species which neatly balances considerable selectivity in feeding against tight budgeting of energy spent in travel. It emerges that the $\mathrm{BCI}$ howlers preferentially feed on relatively clumped fruit and flower sources when available and complement their diet by selectively feeding almost exclusively on young leaves (which, as a rule, contain more protein and less cell-wall material than mature leaves). Seasonal items are particularly important and the howler modifies its feeding pattern over the annual cycle, feeding most on leaves (including a small proportion of mature leaves) when other items are scarce. Despite its concentration on food items with a spatially and temporally patchy distribution, Alouatta is a relatively sluggish primate, resting for about $65 \%$ of daylight hours. It seems to save energy by travelling directly to a few "pivotal" trees (usually fruit-sources) each day, picking up the rest of its food with little extra travel. A wide range of different plant species (at least 73 per troop) is exploited and the overall effect is to limit travel distance to the low level of $443 \mathrm{~m}$ per day. In line with many other primate species, the howler is most active, and has the most diversified diet, during the season of greatest availability of preferred foods. Surprisingly, although the vegetation on $\mathrm{BCI}$ is dense tropical rainforest, there is a marked seasonal pattern (consistent from year to year). Still more surprisingly, it is the "dry" season on BCI that is apparently most favourable in terms of availability of preferred foods of the howlers.

A brief review cannot do justice to the theoretical scope and importance of this monograph. In addition to the central theme of foraging strategy, the text broaches a number of important areas such as the relation between social organization and feeding ecology, the coevolution of plants and animals and the evolution of plant secondary compounds as defence systems - which are at the spearhead of current primate field studies. This is an outstanding book, with only a few minor shortcomings (for example inadequate discussion of problems of bias in data collection and insufficient integration of the results with information from other quantitative studies of howlers and other primates), and it deserves to be widely read by zoologists, primatologists and anthropologists. As a companion volume to Pierre Charles-Dominique's Ecology and Behaviour of Nocturnal Prosimians (1977), Milton's monograph sets a very high standard indeed for any further books on this topic from Columbia University Press.

R.D. Martin is Reader in Physical Anthropology at University College and Visiting Professor in Zoology at Birkbeck College, University of London. 